\title{
Preliminary Results: Exploring Why Some Families Living in Proximity to Wind Turbine Facilities Contemplate Vacating Their Homes-A Community-Based Study
}

\author{
Carmen M. Krogh ${ }^{1}$, Robert Y. McMurtry ${ }^{2}$, Anne Dumbrille ${ }^{3}$, Debra Hughes ${ }^{4}$, Lorrie Gillis ${ }^{5}$ \\ ${ }^{1}$ Magentica Research Group, the Board of Directors, Killaloe, Canada \\ ${ }^{2}$ Schulich School of Medicine and Dentistry, Western University, London, Ontario, Canada \\ ${ }^{3}$ CCSAGE Naturally Green (County Coalition for Safe and Appropriate Green Energy), Picton, Ontario, Canada \\ ${ }^{4}$ Independent, West Lincoln, Canada \\ ${ }^{5}$ Independent, Flesherton, Canada \\ Email: *carmen.krogh@gmail.com
}

How to cite this paper: Krogh, C.M., McMurtry, R.Y., Dumbrille, A., Hughes, D. and Gillis, L. (2020) Preliminary Results: Exploring Why Some Families Living in Proximity to Wind Turbine Facilities Contemplate Vacating Their Homes-A Community-Based Study. Open Access Library Journal, 7: e6118.

https://doi.org/10.4236/oalib.1106118

Received: January 29, 2020

Accepted: February 18, 2020

Published: February 21, 2020

Copyright $\odot 2020$ by author(s) and Open Access Library Inc.

This work is licensed under the Creative Commons Attribution International License (CC BY 4.0).

http://creativecommons.org/licenses/by/4.0/

(c) (i) Open Access

\begin{abstract}
In Ontario, Canada, between 2006 and the end of 2016, government records provided by the former Ontario Ministry of Environment and Climate Change documented that neighbors living near industrial wind turbine (IWT) facilities filed 4574 noise complaints/incident reports. In some cases, these records also included occurrences of adverse health effects being experienced by some of those living near the IWT facilities [1]. The risk of harm associated with living near IWT energy facilities is controversial and reported globally [1] [2] [3] [4]. Some families have been billeted by, or negotiated financial agreements with wind energy developers [2], and some took the step to vacate/abandon their homes [2] [3] [4] while others have felt forced to do so [3] [4]. While the action of vacating/abandoning a family home is internationally reported [1] [2] [3] [4] research about these occurrences is limited. Utilizing the Grounded Theory (GT) methodology, an ethics approved community-based study was conducted to investigate these occurrences. Participants in the study included those who had vacated/abandoned their homes in the past, or at the time of the interview were contemplating to do so, or decided to remain. Between October 2017 and January 2018, sixty-seven (n-67) consenting participants were interviewed. This article presents preliminary results which will be augmented by additional submissions to peer reviewed scientific journals for their consideration for publication.
\end{abstract}

\section{Subject Areas}

Civil Engineering 


\section{Keywords}

Wind Turbines, Grounded Theory, Personal Interviews,

Vacated and Abandoned Homes, Adverse Health Effects

\section{Objectives and Methods}

\subsection{The Research Questions}

To gain an understanding of why some families living in proximity to an IWT facility contemplated vacating their homes, several research questions were identified:

1) What are the particular circumstances which influenced whether to vacate or not vacate a family home?

2) How did families arrive at their decision?

3) Were there consequences related to their decision?

4) Did these circumstances influence physical, mental and social well-being?

\subsection{Study Objectives}

The objectives of the study were to:

1) Explore the events which influenced families living within $10 \mathrm{~km}$ from industrial wind energy facilities to vacate/abandon their homes, contemplating to do so, or remain in their homes;

2) Gain an understanding of the outcomes related to these housing decisions; and

3) Generate a substantive theory associated with the vacated/abandoned home topic.

\subsection{The Grounded Theory Methodology}

The study employed a qualitative research methodology—specifically the Grounded Theory (GT) approach which included the four-phase process proposed by Castillo-Montoya, 2016:

1) Ensuring interview questions align with research questions;

2) Constructing an inquiry-based conversation;

3) Receiving feedback on interview protocols, and

4) Piloting the interview protocol [5].

Before proceeding with the study, the four-phase process was reviewed and incorporated into the vacated home study design.

The following is a brief overview of the methodology employed. This overview will be expanded in a subsequent article.

\section{Methods Overview}

The ethics approval process included provisions such as: the invitation to participate; participant inclusions/exclusions; materials such as the informed consent process including descriptions of the study's purpose and risk/benefits, details relating to the personal (face-to-face) interview, the study process, data own- 
ership and protection of the confidentiality of personal information. Participants were also advised at any time, even if they had signed a consent form, they could decline to answer any question and could change their mind and withdraw participation in the study. If the family did not wish to continue participating in the study, all collected data would be immediately destroyed. Once these points were explained to participants, the consent form was signed by the participants and the interviewer. Both participants and interviewers received a signed copy for their records prior to conducting the interview.

In addition to the requirement to ensure participants were fully informed prior to their participation, they were advised of the intention to submit one or more articles for publication in peer reviewed scientific journals.

If consent were given by the participant(s), the interviewer recorded the interview and a DVD copy was provided to the participant(s) at the conclusion of the interview. In the absence of consent for the audio recording, it was intended the interviewer would take detailed notes; however, all participants consented to the interview being recorded.

Audio files were transcribed to text files by a transcription service (https://gotranscript.com/). NVivo 12 software for qualitative research was leveraged to facilitate coding and data analysis (http://www.qsrinternational.com).

Trained interviewers conducted face-to-face interviews with few exceptions. Due to weather conditions, travel distances and schedules, a few interviews were conducted by telephone.

A brief questionnaire collected background and demographic information. With the completion of the questionnaire, depending on the whether the participant had vacated their home in the past or was contemplating doing so, the interviewer initiated the discussion by asking:

Can you discuss the events that led you to decide to vacate your home?

Or:

Can you discuss the events that led you to think about vacating your home?

All collected data were protected and stored in a secure and locked location. During the tabulation of the collected information, access to all of the collected data including the audio recordings and demographic questionnaires were limited to study investigators.

\section{Invitation to Participate}

A target of twenty-five (n-25) participants was established. However, due to the interest in this study, within a few weeks of its announcement sixty-seven (n-67) participants volunteered to be interviewed. This represents an exceedance of $272 \%$.

In addition to the 67 participants, 98 (n-98) family members were living in the family homes for a total of 165 (n-165). Some participants indicated concerns that others such as their children, grandchildren, relatives, visitors and guests, had experienced a variety of adverse health effects when in the family home. While these reports have not been specifically tabulated in the study findings, some of these observations will be presented in future articles. 


\section{Data Analysis}

The GT iterative process identified numerous themes and sub-themes which evolved as the interviews progressed. Following the interview of the sixty-seventh participant, theoretical saturation was reached and the invitation to participate was closed.

Research indicates a coding paradigm consisting of 5 elements could assist with data analysis by "suggesting what to look for when coding" [6]. Data analysis indicated a relationship with this coding paradigm. This approach was considered appropriate for the vacated home study and its structure adopted.

Table 1: Five elements and their application to the vacated/abandoned home study describes the 5 elements proposed by Rose et al., 2015 and their application to the vacated home study.

Table 1. Five elements and their application to the vacated/abandoned home study.

\begin{tabular}{cc}
\hline Proposed by Rose et al., $2015^{1}$ & $\begin{array}{l}\text { Why some families living in proximity } \\
\text { to wind turbine facilities contemplate }\end{array}$ \\
vacating their homes
\end{tabular}

\section{Element 1}

The central phenomenon that is the focus of the study.

Element 2

The causal conditions that contribute to the phenomenon.

\section{Element 3}

The context in which the phenomenon is embedded.

\section{Element 4}

The actions and interactions taken by people in response to the phenomenon.

\section{Element 5}

The consequences of those actions and interactions.

\section{Element 1}

The central phenomenon/event is the siting of an IWT facility within $10 \mathrm{~km}$ of rural family homes.

\section{Element 2}

The causal conditions are described in the themes and sub-themes such as environmental interference, altered perceptions and living conditions which participants associated with an operational IWT facility within $10 \mathrm{~km}$ of their family home.

\section{Element 3}

The context is embedded in a government policy with research participants becoming informed and taking action through various processes such as filing complaints and participating in legal/judicial processes.

\section{Element 4}

The actions and interactions taken resulted in participants contemplating housing decisions such as: vacating/abandoning a family home; contemplating to do so; preemptively vacating; periodically and intermittently vacating; or deciding to remain.

\section{Element 5}

The consequences of these actions and interactions taken in Element 4 include participants' sentiments and expressions of an "aftermath" such as: profound losses; effects related to social justice, rights, personal security; grief, displacement, anger, bitterness, mistrust, stress and anxiety; financial losses and hardship and impact on employment; and effects on relationships.

${ }^{1}$ Susan Rose, Nigel Spinks \& Ana Isabel Canhoto, 2015. Chapter 6: Management Research: Applying the principles( $\odot$ 2015. Figure 2 Coding paradigm (adapted from Corbin and Strauss 1990, Strauss and Corbin 1998, Corbin and Strauss 2008). 


\section{Results: Highlights and Outcomes}

While the study methodology did not include a structured survey instrument or a research question specific for collecting health symptoms, comments about health arose spontaneously and consistently across participants. This factor was considered by participants as the impetus for decisions on housing choices. Participant data supported that the central phenomenon/event was the siting of an IWT facility within $10 \mathrm{~km}$ of rural family homes (Element 1 ) which participants associated with (Element 2) - the causal conditions of environmental interference, altered living conditions and adverse health effects or awareness of its potential (n-67). This relationship will be expanded in future articles.

\subsection{Diagnosed Medical Conditions and Diseases}

Some participants voluntarily provided documentation of their medical conditions and diseases which were diagnosed by their physicians and physician specialists. These diagnoses included conditions which existed prior to living near a wind energy facility (i.e. a pre-existing condition). While IWT-specific research associated with pre-existing medical and disease conditions is lacking, there was concern that these could potentially be exacerbated by living near IWT facilities. This topic will be discussed in a future article.

\subsection{Vacated/Abandoned Their Homes in the Past}

Twenty-eight (n-28) of the 67 study participants had vacated/abandoned their homes in the past.

Example 1

... they started up the wind turbines around us... As soon as they were all up and running we immediately suffered from sleep disturbance ... it was the loud noise, it was like a whooshing noise you could hear it inside the house and it was vibration which was terrible... you could hear and feel it.

Example 2

I had symptoms of electrical sensitivity which I had not experienced that way in the house before. The only thing that had changed in the environment was the turbines going up.

Example 3

... the first instance was the building of the [location protected] wind turbines ... my whole intestinal tract started to quiver ... As the days and weeks progressed, I found that I was having difficulty with my ears...

\subsection{Contemplating to Vacate/Abandon Their Homes}

Thirty-one (n-31) of the 67 study participants were contemplating to vacate/abandon their homes.

Example 1

... when it was announced that wind turbines were coming ... P m concerned about my health and the fact that I developed a condition since the turbines 
started that is related to-what's the term when your body fights against itself-autoimmune disease since the turbine started.

Example 2

Initially, when neighbors decided to put turbines in. Because of my knowledge and my previous studying about the possible health effects, and the effects to animals I already thought before they turned the soil that there may be issues coming ahead, and that was three years ago.

Example 3

We started having this ringing in our ears, buzzing, like a buzzing noise in our ears ... we had three different electricians come ... one electrician told us, "This is a frequency coming into your home. You need to put a for sale sign up and get out of here."

\subsection{Deciding to Remain}

Four participants (n-4) decided to remain.

Example 1

I know people that have vacated their homes because of the side effects ... If it does become an issue, we certainly would. A health issue or affecting either one of us, we certainly would.

Example 2

We pulled the plug on building a new home ... That was because as some of the information that I had learned made both my husband and I were wary of building a new home close to wind turbines...

\subsection{Preemptively Vacated/Abandoned Their Homes}

Four participants (n-4) expressed awareness of the potential risk of adverse health effects and took preemptive action and vacated/abandoned their homes prior to an IWT facility initiating operations.

Example 1

Basically knowing the health issues ... it just solidified it more and more for us then, we just need to move away from all of these. Not just from turbines but from the strife.

Example 2

... we became involved in the issues with turbines in the early 2000s more because the turbines were going to be put in around us ... Probably the first real concern was the health issues, some of the things that we had seen.

\subsection{Periodically and/or Intermittently Vacating/Abandoning a Family Home}

As the interviews progressed, the iterative process led to additional findings which participants associated with an operating IWT facility. Comments revealed that some participants intermittently/temporarily vacated their homes to obtain temporary and/or partial relief from the occurrence of adverse health effects. 


\subsubsection{Vacated/Abandoned Their Homes in the Past}

\section{Example 1}

When I left my home, whether that be for [location protected] in the morning or quite often in the middle of the night, I left my home and then I slept in my vehicle away from the turbines and I would recover from all these symptoms.

\section{Example 2}

Leaving the house four to five nights all the time, sleeping in my vehicle in the cold in the winter. I would be in my vehicle five, six hours during the night away from the turbines by myself.

Example 3

... we were not permanent residents of our homes. We were constantly leaving when the symptoms and the effects of the wind project on us were so great we didn't stay.

\subsubsection{Contemplating to Vacate/Abandon Their Homes}

Example 1

We left for three months ... we went away ... just to try it. Just to see what it was like and it did help. Since then, it helped last year, we have a place in [location protected] that we' re going to go to get away from the turbines because we just can't stand the turbines.

Example 2

We've left home many times for the day just because of the noise here ... I remember one morning ... it was shortly after seven in the morning and we left home that day because we couldn't stand it ...

Example 3

... one winter was just so bad at times; we just kept going for drives to get out of the house for a bit, just to try to get some relief ...

The motivation to periodically/intermittently vacate a home will be discussed in a future article.

\section{Conclusions}

Of the 67 (n-67) study participants: 28 (n-28) had vacated/abandoned their home; 31 (n-31) were contemplating to do so; $4(n-4)$ preemptively vacated their home before the facility started operating; and 4 (n-4) decided to remain. Figure 1 illustrates the home status of the 67 participants at the time of the interviews.

Some of the study participants' physicians, including physician specialists diagnosed medical and disease conditions, some of which were diagnosed prior to the presence of an IWT facility, i.e., a pre-existing condition. While IWT-specific research relating to such pre-existing conditions is lacking, some participants expressed concern regarding a potential exacerbation of their symptoms. It is proposed the potential risk factors associated with pre-existing medical/disease conditions should be investigated as soon as possible. 


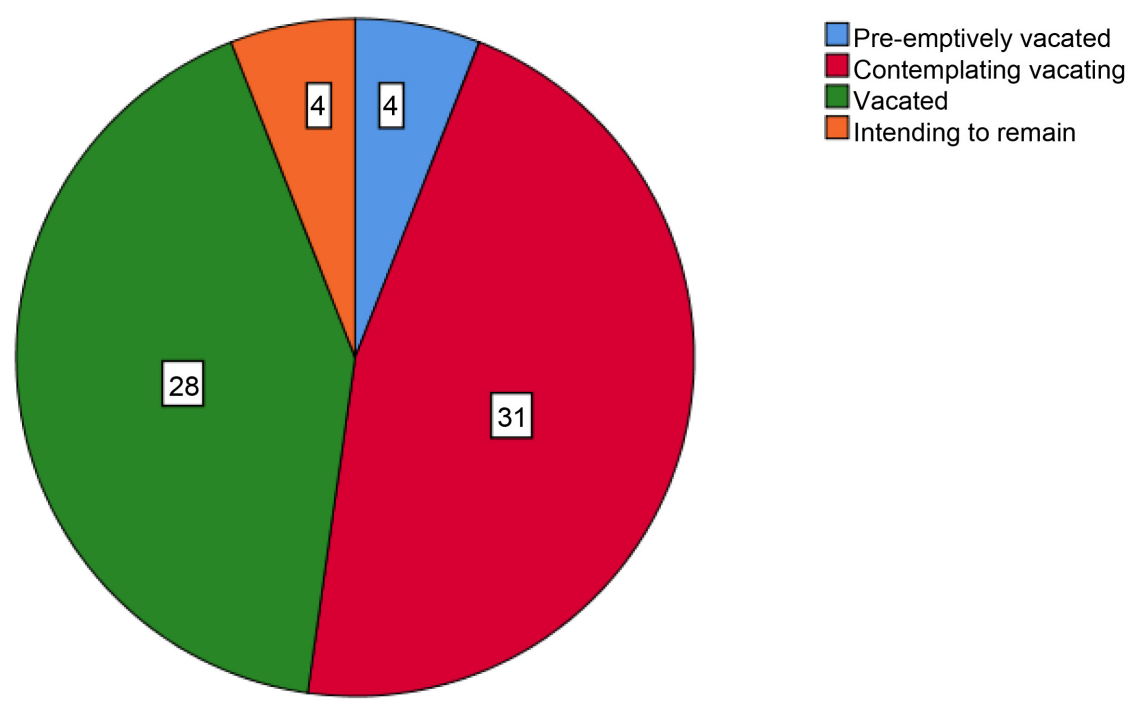

Figure 1. Home status at the time of interview.

The iterative interview process led to additional findings which participants associated with the start up of an IWT facility. Some participants from both categories of having vacated a home in the past or contemplating to do so had periodically and intermittently vacated their homes during the day and/or night. Both groups indicated the reason for this was to obtain temporary and/or partial relief from the occurrence of adverse health effects.

Data analysis supports the theory that housing decisions were motivated by the presence of an IWT facility within $10 \mathrm{~km}$ of family homes and the participants' observations of an association or a potential risk of adverse health effects.

These preliminary results will be augmented by additional submissions to peer reviewed scientific journals for their consideration for publication. Topics will include results of the data analysis relating to the elements described in Table 1. Five elements and their application to the vacated/abandoned home study.

\section{Conflicts of Interest}

The authors declare no conflicts of interest regarding the publication of this paper.

\section{Funding}

MAGENTICA RESEARCH GROUP.

Not for Profit (Corporations Canada).

Partially funded by private donation.

\section{Ethics Review}

Chesapeake Research Review, LLC (“Chesapeake IRB”).

Note: Chesapeake Research Review, LLC (“Chesapeake IRB”) and Schulman Associates Institutional Review Board, Inc. ("Schulman IRB") have merged to create Advarra, Inc. (“Advarra IRB”). 


\section{Acknowledgements}

We wish to acknowledge everyone who participated in this important research and who shared their experiences when living in proximity to wind energy facilities. We thank them for welcoming our interviewers into their homes. Without their participation and their willingness to describe the profound impact on their lives when contemplating vacating a family home, this research would not have taken place. Our research team is honored to have been given the opportunity to bring these findings forward. We thank our interviewers, who volunteered their time and travelled considerable distances in order to conduct the interviews and those who provided the funding to make this study possible.

\section{References}

[1] Krogh, C.M., Wilson, E.J. and Harrington, M.E. (2019) Wind Turbine Incident/Complaint Reports in Ontario, Canada: A Review-Why Are They Important? Open Access Library Journal, 6, e5200.

[2] Jeffery, R.D., Krogh, C.M. and Horner, B. (2014) Industrial Wind Turbines and Adverse Health Effects. The Canadian Journal of Rural Medicine, 19, 21-26.

http://www.ncbi.nlm.nih.gov/pubmed/24398354

[3] Krogh, C.M.E. (2011) Industrial Wind Turbine Development and Loss of Social Justice? Bulletin of Science Technology \& Society, 31, 321-333.

http://bst.sagepub.com/content/31/4/321 https://doi.org/10.1177/0270467611412550

[4] Le Coz, E. and Sherman. L. (2017) In the Shadow of Wind Farms. How the Wind Industry Angers Landowners and Divides Communities in Pursuit of Billions of Dollars in Subsidies and Other Incentives. Gatehouse Media.

http://gatehousenews.com/windfarms/home/?skipintro=true?skipintro=true?skipin $\underline{\text { tro=true }}$

[5] Castillo-Montoya, M. (2016) Preparing for Interview Research: The Interview Protocol Refinement Framework. The Qualitative Report, 21, 811-831. https://nsuworks.nova.edu/tqr/vol21/iss5/2/

[6] Rose, S., Spinks, N. and Canhoto, A.I. (2015) Chapter 6: Management Research: Applying the Principles.

https://pdfs.semanticscholar.org/6182/186784d792ed09c4129924a46f9e88869407.pdf 